\title{
Using Cumulative Count of Conforming CCC-Chart to Study the Expansion of the Cement
}

\author{
Dr.Kawa M. Jamal Rashid ${ }^{1}$, Dr.Abdul Rahim Khalaf Rahi ${ }^{2}$ \\ ${ }^{1}$ University of Sylaimanyai College Of Administration and Economics Department Of Statistics ${ }^{2}$ University of \\ Mustanisirya, College Of Administration and Economics Department Of Statistics
}

\begin{abstract}
In this paper, we discuss the need for process improvement and problem that may be faced in controlling high Quality process, we first review some basic concepts in statistical process control and control charts, the focus is on the modification of the traditional control charts, for attributes the cumulative count of conforming (CCC) that is a powerful technique based on counting of cumulative conforming item between nonconforming ones.
\end{abstract}

The CCC chart is very easy to implement, efficient in detecting process changes.

Keywords: Traditional Control Charts, Modification Of Control Chart, The Cumulative Count of Conforming (CCC) chart

\section{Introduction}

Control chart are widely used in industry as a tool to monitor process characteristics. Deviations from process targets can be detected based on evidence of statistical significance.

Statistical process control originated in early twenties when Shewhart (1926) presented ideas of statistical control charts for process characteristics.

The basic principle is that processes are always subject to random variation, which is generally not controllable ordered identifiable. Thus some variation within limits are allowed in order to do this we can plot successively observed process characteristics and make decisions based on what has actually been observed in the long run.

\section{Traditional Control Charts}

Control chart in industry are divided into two main types, variable charts and attribute charts .For variable charts, the process or quality characteristics take on continuous values while for attribute charts the data in this form of discrete counts.

The P-chart is usually used to monitor the proportion nonconforming in a sample the control limits for the $\mathrm{p}$ or $n p-$ Chart can be derived in the following manner, a sample size $\mathrm{n}$ follows the binomial distribution with the parameter $\mathrm{p}$, with mean $n p$ and variance $n p q$

The upper control limit and lower control limit for p-cart given by:

$$
\begin{aligned}
& U C L, L C L=p \pm 3 \sqrt{n p(1-p) / n} \quad \ldots 2-1 \\
& U C L, L C L=n p \pm 3 \sqrt{n p(1-p)} \quad \ldots 2-2
\end{aligned}
$$

And the central limit is:

$C l=p$

And for np-chart, which is used for the monitoring number of non-conforming items in samples of size $\mathrm{n}$, modified limits are as follows:.

$$
\begin{gathered}
U C L, L C L=n p \pm 3 \sqrt{n p(1-p)} \quad \ldots 2-3 \\
C l=n p
\end{gathered}
$$


When the sample size is fixed for all sample the $p$-chart and $n p$-Chart are very similar and the different is only in the scale of the y-axis. Different p-charts can be easily compared as the center line for the process fraction nonconforming level .For $n p$-Chart the center line is affected by the sample size.

When $\mathrm{p}$ or $n p-$ Chart are used it is important to use the appropriate limit, because of the skewness of binomial distribution, the lower limit based on 3 sigma concept may not exist as it is usually a negative value, probability limit should be used when possible .On the other hand, simple modification can be used to obtain better control limit for $\mathrm{p}$ or $n p-$ Chart .

\section{Modification Of Control Chart}

Some improvement made on traditional $\mathrm{p}$ and $n p$-Chart using some transformations so as to achieve high power in detecting $\mathrm{np}$-words shift in a process, with a simple adjustments to the control limits of the p-chart one can achieve equal or even better results form of the available methods are as follows:

\section{3-1 Binomial Q chart:}

By transforming the binomial variable to standard normal variable, a Q-chart (Queensberry 1997),could be drawn, with upper and lower control limits equal to 3 and -3 respectively it is defined by:

$$
u_{i}=\sum_{k=0}^{i}\left(\begin{array}{l}
n \\
p
\end{array}\right) p^{k}(1-p)^{n-k} \quad \ldots 3-1
$$

And

$$
Q_{i}=\phi^{-1}\left(u_{i}\right)
$$

Where $\phi^{-1}$ (.) is the inverse function of the standard normal variable.

Plotting $\phi_{i}$ s on the chart, standard normal control chart with a uniform limit of ( 0 and 1$)$ could be maintained.

\section{3-2 Arcsine p-chart}

This is another nonlinear transformation like in the binomial Q chart with

$$
W_{i}=2 \sqrt{n_{i}}\left[\sin ^{-1}\left(\sqrt{\frac{x_{i}+\frac{3}{8}}{n_{i}+\frac{3}{8}}}\right)-\sin ^{-1}(\sqrt{p})\right] \ldots 3-2
$$

Since $W_{i}$ is the approximately standard normal, a chart could be drawn by plotting $W_{i}$ on the chart with control limits are of 3 and -3 .

\section{3-3 Cornish Fisher expansion method.}

This method having some additional constant values in the three sigma limits using the normal distribution.

Define $\quad Y_{i}=X_{i} / n$ Plotting $\mathrm{Y}_{\mathrm{i}}$ values on the chart it is very easy to detect the changes in $\mathrm{p}$ values with the following control limit

$$
U C L, L C L=P \pm 3 \sqrt{\frac{P(1-P)}{n}}+\frac{4(1-2 P)}{3 n}
$$

When the $\mathrm{p}$ is unknown, they have to be estimated and inserted in the above equations.

It is noted that the above equations could be used for variable sample sizes replacing $n=n_{i}$

\section{3-4 Modified limits for np-Chart}

Ryan and Schwartzman (1997) proposed the following regression equations of $n p$ and $\sqrt{n p}$ and found them accurate. The proposed control limits are. 


$$
\begin{aligned}
U C L & =0.6195+1.00523 n p+2.983 \sqrt{n p} \\
U C L & =2.9529+1 . .01956 n p-3.2729 \sqrt{n p}
\end{aligned}
$$

It is noted that these limits are valid for $(\mathrm{p}<0.03)$.

While assuming the acceptable average run length (ARL), which is the average number of points plotted before an alarm to be (370 .4.).In comparing with the exact limit these limits are less biased in terms of ARL than the Shewhart p-chart.

\section{The Cumulative Count Of Conforming (CCC) Chart}

The Cumulative Count of Conforming (CCC) chart or the count of conforming control chart is first developed in Calvin (1983) to monitor ZD (zero-defects) process, It was further studied and given the name CCC - chart by Goh (1987) the use of ccc type control chart has been further studied by Lucas (1989), Bourke (1991), Xie and Goh (1992) , and Wu et al (1999-2000).

This is a powerful techniques for process control when a larger of consecutive conforming items are observed between two nonconforming ones.

The idea behind the CCC-charts is the fact that the number of conforming items between two consecutive nonconforming ones changes when the fraction nonconforming shifts themselves in near ZD processes because they are usually vary small in the absolute magnitudes.

\section{Setting Up Of The CCC Chart}

The setting of the CCC chart is similar to the generic procedure of the setting up a Shewarthart control chart except that the measurement are the number of conforming items after the last nonconforming one . This count should be plotted only when a new conforming item is observed.

Let $(\mathrm{n})$ be the number of items observed before a nonconforming one is found i.e (n-1) items are conforming followed by $\mathrm{n}^{\text {th }}$ item which is nonconforming .it is clear that this count follows a geometric distribution. The determination of the control limits based on this.

If the probability nonconforming item is equal to $\mathrm{p}$ then the probability of getting ( $\mathrm{n}-1)$ conforming ones following a nonconforming one is:

$$
p(1-p)^{n-1} \mathrm{n}=1,2, \ldots
$$

The mean of geometric distribution with parameter $\mathrm{p}$ which can be used as the center line is :

$$
C L=1 / P
$$

Suppose that the acceptable of false alarm probability is $\alpha$, the UCL and LCL can be determined as:

$$
U C L=\frac{\ln (\alpha / 2)}{\ln (1-p)} \quad \ldots 5-3
$$

And

$$
L C L=\frac{\ln (1-(\alpha / 2))}{\ln (1-p)}
$$

Respectively control charts can be set up by including the control limits and the central line.

If the proportion of non-conforming items a associated with the process is $p$, then the probability that no nonconforming item is found in $\mathrm{n}$ inspected is

$\mathrm{P}($ no nonconforming in $\mathrm{n}$ items $)=(1-\mathrm{P})^{\mathrm{n}}$

This prob. reflects the certainty we have when we judge the process to be out of control when a nonconforming item has been found. Xie and Goh (1992) introduced the concept of certainty level S,

Which is the probability that the process is actually out of control?

The certainty level, S related to false alarm probability when interpreting CCC chart signal. With one side control limit

$$
S=1-\alpha \quad \ldots .5-6
$$
is given as:

The relation between the proportion of nonconforming items $\mathrm{P}$ and number of items inspected $\mathrm{n}$ which

$$
(1-p)^{n}=S
$$


We can now determine the number of conforming items inspected before a nonconforming one is allowed for the process to still be considered in control, this can be calculated as:

$$
n=\frac{\ln S}{\ln (1-p)}
$$

For practical applications a decision graph can be constructed to facilitate decisions on the state of control of process whenever nonconforming item is observed.

\section{Application}

The important factor that describe the quality of the cement is the (expansion of the cement - EXP) where the standard value of this feature is less than $10 \mathrm{~mm}$. A set of CCC data is shown in table (6-1)

Table (6-1) shows that the cumulative data. With acceptable value of EXP is $<10$

Table (6-1) Uncensored -CCC_EXP data

\begin{tabular}{|c|c|c|c|c|c|c|c|}
\hline No & CCC & No & CCC & No & CCC & No & CCC \\
\hline 1 & 10 & 11 & 0 & 21 & 1 & 31 & 5 \\
\hline 2 & 18 & 12 & 0 & 22 & 0 & 32 & 4 \\
\hline 3 & 0 & 13 & 12 & 23 & 0 & 33 & 17 \\
\hline 4 & 0 & 14 & 3 & 24 & 0 & 34 & 3 \\
\hline 5 & 6 & 15 & 0 & 25 & 0 & 35 & 1 \\
\hline 6 & 0 & 16 & 1 & 26 & 41 & 36 & 0 \\
\hline 7 & 0 & 17 & 0 & 27 & 45 & 37 & 3 \\
\hline 8 & 8 & 18 & 41 & 28 & 51 & 38 & 3 \\
\hline 9 & 6 & 19 & 20 & 29 & 13 & 39 & 0 \\
\hline 10 & 31 & 20 & 0 & 30 & 4 & 40 & 0 \\
\hline & & & & & & 41 & 1 \\
\hline
\end{tabular}

Calculate the upper and lower of CCC-EXP of table (6-1) by using the equation (5-3 and 5-4),(44.9, 0.156) respectively in Fig(6-1) shows the corresponding CCC chart is displayed.

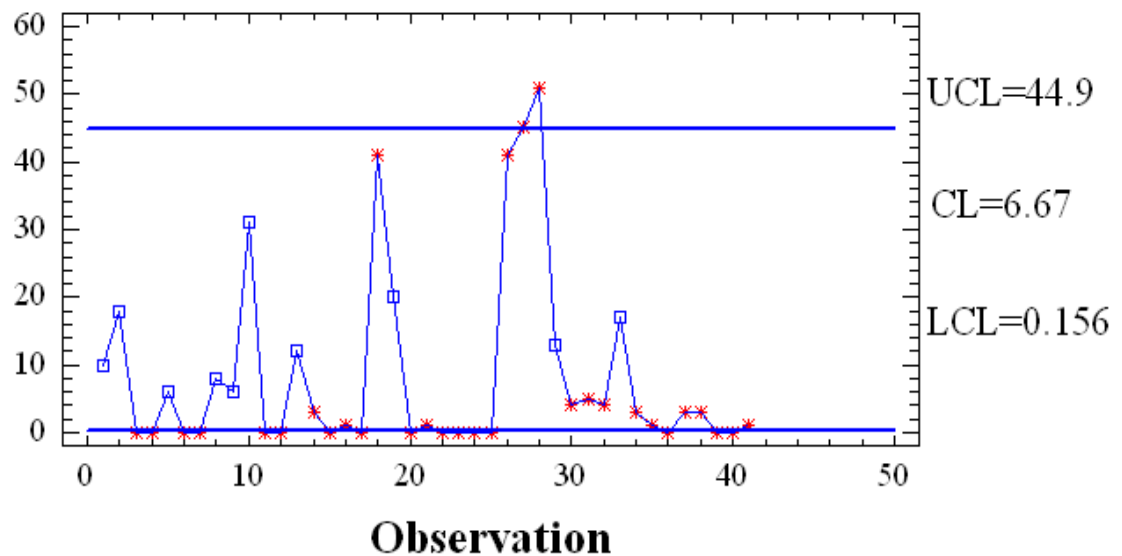

Fig (6-1) Acceptance chart of CCC-EXP data

In this case two points out of the upper control limit and (16) points are out of the lower control limit and the process in out of the control. 
Table (6-2) shows that the frequency of occurrence of the 17 unique the classes are sorted according to the counts, with the most frequently occurring class first. The highest class is 0 with a count of 16, which represents $39.0244 \%$ of the total, and Fig (6-2) shows the Pareto chart.

Table (6-2) Pareto Cumulative Frequencies of CCC-EXP data

\begin{tabular}{|c|c|c|c|c|c|}
\hline $\begin{array}{c}\text { Class } \\
\text { Label }\end{array}$ & Rank & Count & $\begin{array}{l}\text { CuSum } \\
\text { Score }\end{array}$ & $\begin{array}{l}\text { Percent } \\
\%\end{array}$ & $\begin{array}{l}\text { Cusum } \\
\text { Percent } \\
\%\end{array}$ \\
\hline 0 & 1 & 16 & 16 & 39.02 & 39.02 \\
\hline 3 & 2 & 4 & 20 & 9.76 & 48.78 \\
\hline 1 & 3 & 4 & 24 & 9.76 & 58.54 \\
\hline 41 & 4 & 2 & 26 & 4.88 & 63.41 \\
\hline 6 & 5 & 2 & 28 & 4.88 & 68.29 \\
\hline 4 & 6 & 2 & 30 & 4.88 & 73.17 \\
\hline 51 & 7 & 1 & 31 & 2.44 & 75.61 \\
\hline 45 & 8 & 1 & 32 & 2.44 & 78.05 \\
\hline 31 & 9 & 1 & 33 & 2.44 & 80.49 \\
\hline 20 & 10 & 1 & 34 & 2.44 & 82.93 \\
\hline 18 & 11 & 1 & 35 & 2.44 & 85.37 \\
\hline 17 & 12 & 1 & 36 & 2.44 & 87.8 \\
\hline 13 & 13 & 1 & 37 & 2.44 & 90.24 \\
\hline 12 & 14 & 1 & 38 & 2.44 & 92.68 \\
\hline 10 & 15 & 1 & 39 & 2.44 & 95.12 \\
\hline 8 & 16 & 1 & 40 & 2.44 & 97.56 \\
\hline 5 & 17 & 1 & 41 & 2.44 & 100 \\
\hline Total & & 41 & & & \\
\hline & & & & & \\
\hline
\end{tabular}

Fig (6-2) Pareto Chart OF CCC-EXP data Transformation:

Pareto Chart for Col_ 1

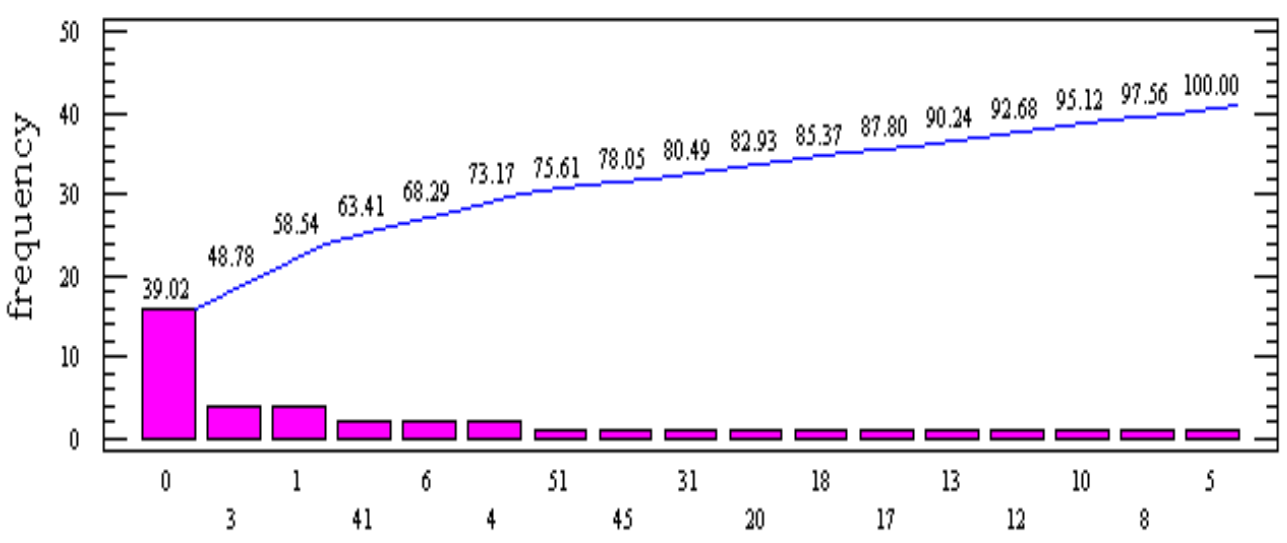

6-1 Charts based on Transformation 
The transformation is useful when the distribution is non-normal which is the case for geometric distribution under the transformation, the data become normally distributed. There are a number of transformations available, few of there are shows below:

1-The double square root Transformation

A double square root for the (fourth root) is a simple transformation by:

$$
Y=X^{(0.25)}, \quad \mathrm{x} \geq 0
$$

The below table Show that the illustrate some possible transformation approaches.

Table (6-3) Transformations of CCC-EXP data

\begin{tabular}{|c|c|c|c|c|c|c|c|}
\hline$Y=X^{(0.25)}$ & $\operatorname{Ln}(\mathrm{x})$ & $Y=X^{(0.25)}$ & $\operatorname{Ln}(\mathrm{x})$ & $Y=X^{(0.25)}$ & $\operatorname{Ln}(\mathrm{x})$ & $Y=X^{(0.25)}$ & $\operatorname{Ln}(\mathrm{x})$ \\
\hline 1.778 & 2.303 & 0 & & 1.000 & & 1.495 & 1.609 \\
\hline 2.060 & 2.890 & 0 & & 0 & & 1.414 & 1.386 \\
\hline 0 & & 1.861 & 2.485 & 0 & & 2.031 & 2.833 \\
\hline 0 & & 1.316 & 1.099 & 0 & & 1.316 & 1.099 \\
\hline 1.565 & 1.792 & 0 & & 0 & & 1.000 & 0 \\
\hline 0 & & 1.000 & 0 & 2.530 & 3.714 & 0 & 1.316 \\
\hline 0 & & 0 & & 2.590 & 3.807 & 1.316 & 1.099 \\
\hline 1.682 & 2.079 & 2.530 & 3.714 & 2.672 & 3.932 & 0 & \\
\hline 1.565 & 1.792 & 2.115 & 2.996 & 1.899 & 2.565 & & \\
\hline 2.360 & 3.434 & 0 & & 1.414 & 1.386 & & \\
\hline & & & & & & & \\
\hline
\end{tabular}

In Fig (6-3) the corresponding CCC chart is displayed in this case , all points are within the control limits and the process is in control compare with fig.(6-2)

$X$ Chart for Transformations

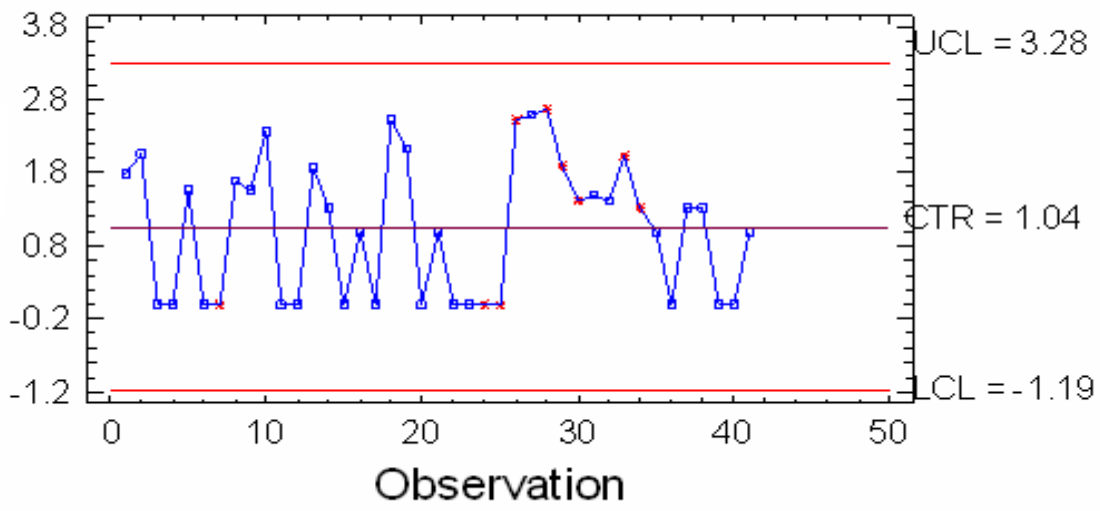

Fig(6-3) The of Transformation data chart of CCC_EXP

\section{6-2 Quesenberry's Q-Transformation}

Using $\mathrm{Q}^{-1}$ to denote the inverse function of standard normal distribution, define

$Q_{i}=-Q^{-1}\left(u_{i}\right)$

where

$$
u_{i}=F\left(x_{i}, p\right)=1-(1-p)^{x_{i}} \quad \text { For } i=1,2,3, \ldots Q_{i}
$$

Will approximately follow the standard normal distribution, and the accuracy improves as $\mathrm{p}$ approaches zero. 
Table (6-4) shows the data of Queensberry's Transformation of CCC-EXP data

\begin{tabular}{|c|c|c|c|}
\hline $\mathrm{Q}_{\mathrm{i}}$ & $\mathrm{Q}_{\mathrm{i}}$ & $\mathrm{Q}_{\mathrm{i}}$ & $\mathrm{Q}_{\mathrm{i}}$ \\
\hline 0.2327 & 2.326 & 0.7507 & 0.7621 \\
\hline-0.2611 & 2.326 & 2.326 & 0.9385 \\
\hline 2.326 & 0.1257 & 2.326 & -0.2224 \\
\hline 2.326 & 1.0494 & 2.326 & 1.0494 \\
\hline 0.628 & 2.326 & 2.326 & 0.7507 \\
\hline 2.326 & 0.7507 & -1.165 & 2.326 \\
\hline 2.326 & 2.326 & -1.2873 & 1.0494 \\
\hline 0.4316 & -1.165 & 1.4532 & 1.0494 \\
\hline 0.628 & -0.3836 & 0.0426 & 2.326 \\
\hline-0.831 & 2.326 & 0.9002 & 2.326 \\
\hline & & & 0.7507 \\
\hline
\end{tabular}

\section{XChartfor Quesenberry}

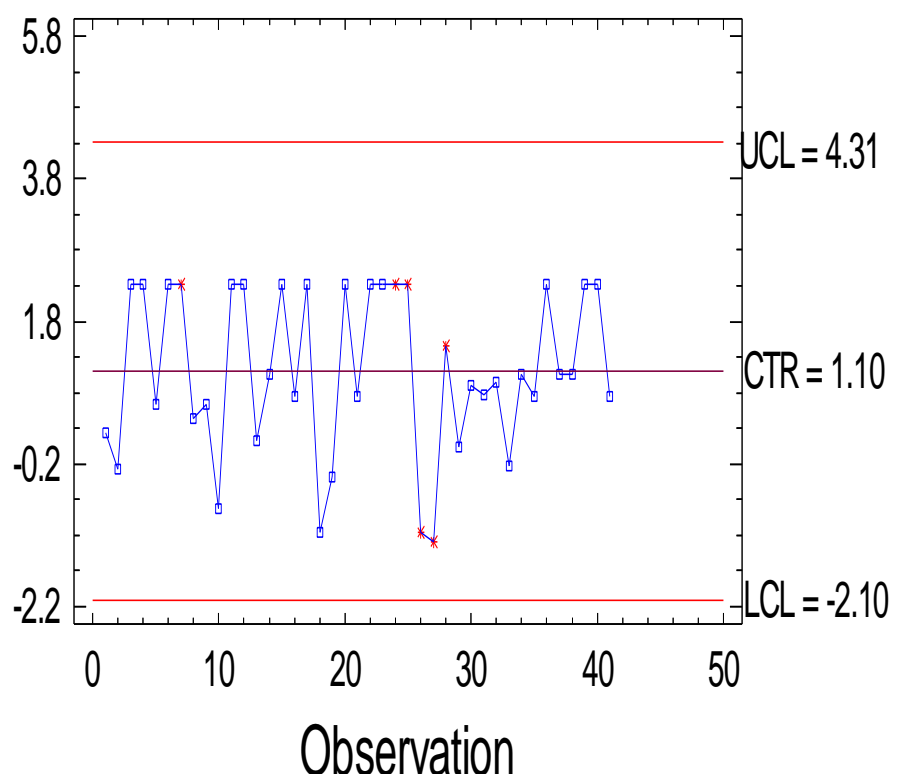

Fig (6-4) the individual chart of CCC_EXP data with Q- Transformation

From above chart of Q- Transformation, it shows that all points are within the control limits and the process is in control.

\section{6-3 finding the Sample Size at different fractional non conforming level at certainly level of $\mathbf{S}$}

To find the Sample Size at different fractional non conforming level at certainly level of $S$ value by using the equation (5-7 and 5-8) and depend on the $\mathrm{P}$, and $\mathrm{S}$ value it seen the result in the below table (6-5). 
We give some numerical values of (n) for some different combinations of values of $\mathrm{p}$ and $\mathrm{S}$. If the cumulated count of conforming item is less than the tabulated value, then the proportion of nonconforming items is higher than with certainty $\mathrm{S}$.

This table can be used to determine the value of certainty level (s) for a given level of proportion nonconforming $\mathrm{P}$, as well as determine the value of ( $\mathrm{p}$ ) for given level of certainty and see if it is higher than the acceptable level for the process .Fur there more for given ( $p$ and $s$ ), determine the minimum number of conforming item that must have been observed before a nonconforming one can be tolerate ie, the process can still be deemed under control even one nonconforming item has been observed.

Table (6-5) Sample Size at different fractional non conforming level

\begin{tabular}{|c|c|c|c|}
\hline P value & $\begin{array}{c}\text { If } \\
S_{1}=0.90 \\
n\end{array}$ then & $\begin{array}{l}\quad \text { If } \\
S_{2}=0.95 \\
\text { then } n\end{array}$ & $\begin{array}{r}\text { If } \\
S_{3}=0.98 \\
n\end{array}$ then \\
\hline 0.001 & 105.308 & 51.268 & 20.193 \\
\hline 0.002 & 52.628 & 25.621 & 10.091 \\
\hline 0.003 & 35.067 & 17.072 & 6.724 \\
\hline 0.004 & 26.287 & 12.798 & 5.041 \\
\hline 0.005 & 21.019 & 10.233 & 4.030 \\
\hline 0.006 & $\mathbf{1 7 . 5 0 7}$ & 8.523 & 3.357 \\
\hline 0.007 & 14.999 & 7.302 & 2.876 \\
\hline 0.008 & 13.117 & 6.386 & 2.515 \\
\hline 0.009 & 11.654 & 5.674 & 2.235 \\
\hline 0.01 & 10.483 & 5.104 & 2.010 \\
\hline 0.011 & 9.525 & 4.637 & 1.826 \\
\hline 0.012 & 8.727 & 4.249 & 1.673 \\
\hline 0.013 & 8.052 & 3.920 & 1.544 \\
\hline 0.014 & 7.473 & 3.638 & 1.433 \\
\hline 0.016 & 6.532 & 3.180 & 1.253 \\
\hline 0.018 & 5.801 & 2.824 & 1.112 \\
\hline 0.02 & 5.215 & 2.539 & 1.000 \\
\hline
\end{tabular}




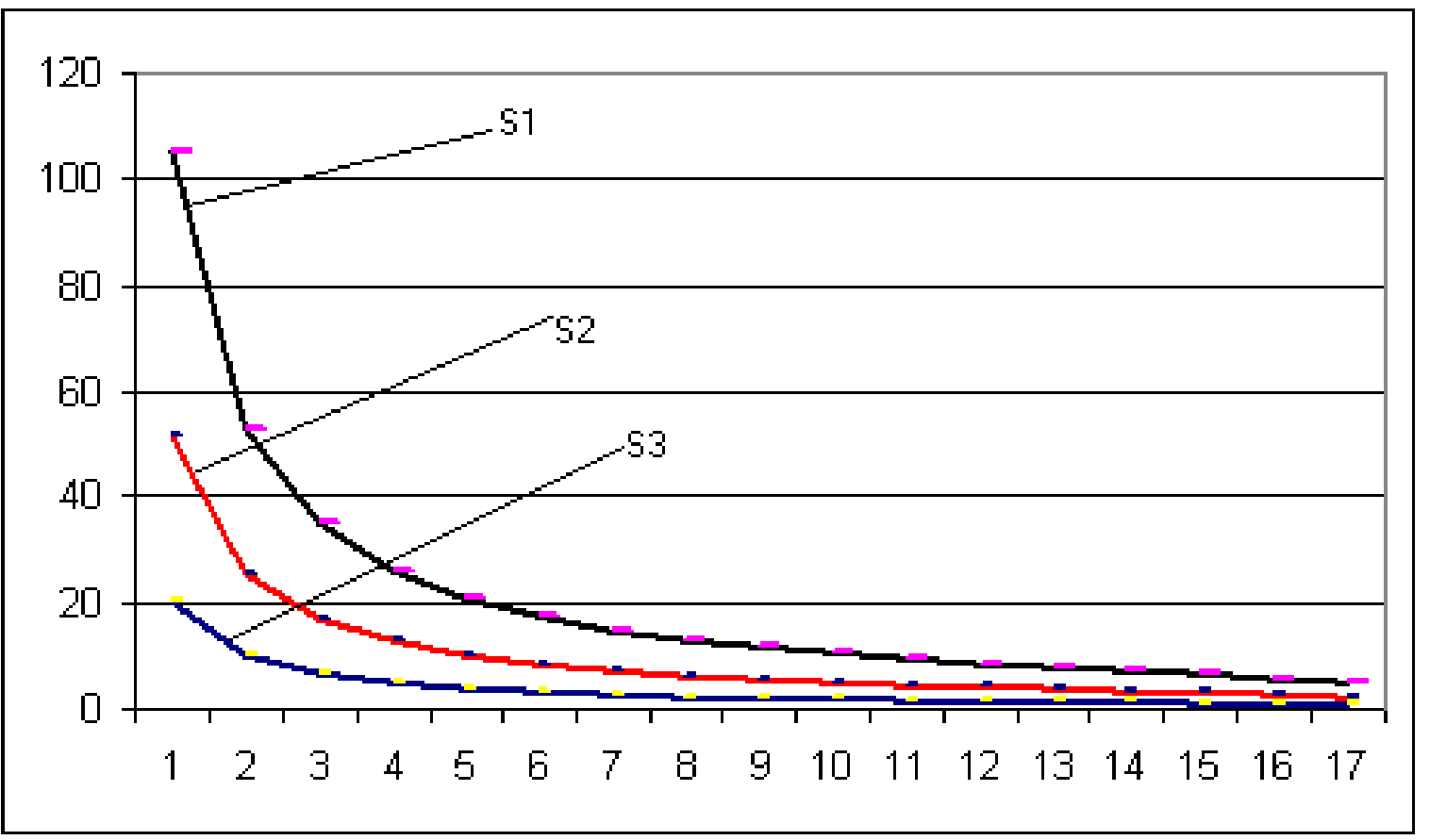

Fig( 6-5) A decision graph for the stat of control of a process

Fig (6-5) Show the chart of the sample size of different fractional non conforming level at certainty level, s Number Of -non- conforming items percent nonconforming.

Since usually $\mathrm{p}<1$ for high- quality products, a good approximation of Eq.(5-7) is

$$
S=e^{n p} \quad, \quad P \leq 1
$$

Hence, for high-quality products, we have an expontional relation between the number of inspected items $\mathrm{n}$ and the confidence that the process is out of control when the single nonconforming item is found.

Then exponential relation is very convenient to use from another point of view. By taking logarithms, we can show that the relation is equivalent to .

$$
\operatorname{Ln} n+\operatorname{Ln} p=\operatorname{Ln}(-\operatorname{LnS})
$$

\section{Conclusion}

1- The CCC-chart is easy to use and more sensitive to short-term drifts in process parameter as well as enable its user to judge the state of control.

2- The decision graph can be used for combination of proportion non-conforming, $\mathrm{P}$ and certainly level, $\mathrm{S}$ to determine the minimum number of conforming item.

3- Under the transformations either Q-transformation or a double square root, the data of Expansion become normally distribution and the accuracy improves as well as the process is improved also (all points are within control limits).

\section{Reference}

[1]. Anscombe F,FJ (1948)The transformation of Poisson, Binomial, and negative binomial data

[2]. Bourke,P.D(1991)Detecting shift in fraction nonconforming using run length control charts with $100 \%$ inspection.

[3]. Bourke,P.D(2001) Sample size and Binpmial Cusum control chart the case of $100 \%$ inspection

[4]. Crossley, M.L. (2000) The Desk Reference of Statistical Quality Methods, ASQ Press, Milwaukee, WI, USA.

[5]. Ishikawa, K. (1986) Guide to Quality Control, Asian Productivity Association, Tokyo, Japan.

[6]. Johlan Oakland Sixth edition 2008 Statistical Process Control

[7]. James R.; Koronacki T.Jacek 2002 by Chapman \& Hall/CRC STATISTICAL PROCESS CONTROL The Deming Paradigm and Beyond ,second edition

[8]. Kemp, K.W. (1962) Applied Statistics, Vol.11, pp.16-31, 'The use of cumulative sums for sampling inspection schemes.'

[9]. Xie, TN Goh, V Kuralmani (2002) Statistical Model and Control Chart for High- Quality Process.

[10]. Oakland, J.S.(2000)Total Quality Management-Textand Cases, 2nd Edition, Butterworth -Heinemann, Oxford, UK. 
[11]. Ognyan Ivanov 2011 APPLICATIONS AND EXPERIENCES OF QUALITY CONTROL

[12]. PETER W. M. JOHN (1990) Statistical Methods in Engineering and Quality Assurance

[13]. Xie, M: Goh T.N and tang X,Y(2000) Data Transformation for geometrically distributed quality characteristics

[14]. Yang, Z.I : Xie M and Goh (2000) Process monitoring of exponentially distributed characteristics through an optimal normalizing transformation. 